\title{
Prevention unstable conditions in the welding process via robotic technological complexes
}

\author{
Dmitry Fominykh ${ }^{1, *}$, Vadim Kushnikov ${ }^{1}$, and Alexander Rezchikov ${ }^{1}$ \\ ${ }^{1}$ Institute of Precision Mechanics and Control, Russian Academy of Sciences, 24, Rabochaya str., \\ Saratov, 410028, Russia
}

\begin{abstract}
The article presents the statement of the problem, models and algorithms for controlling the welding process via robotic technological complexes in conditions of the risk of unstable states. The system dynamics model was used to describe the technological process. As system levels the basic indicators influencing quality of the made production chosen on the basis of experience of operation of the robotic technological complexes are taken. Functional dependences between indicators and their dependences on external factors are determined by approximation of statistical data. The procedure of identification of unstable states for the mathematical model on the example of the Rossler's attractor is developed. An algorithm for preventing the system from falling into unstable states in the process control is proposed
\end{abstract}

\section{Introduction}

When welding steel in robotic technological complexes (RTC) requires a constant assessment of the quality at all stages of the production cycle, including operator control of welding quality during welding, the periodic control by the programmer of the welding parameters, full monitoring of production quality by quality-control (QC) inspector, etc. The lack of control at any stage, for example, due to a deviation in the welding parameters or a insufficient of QC inspectors increases the risk of defective products.

Now various systems of quality control of welding in RTC are developed and applied [1-6]. Mostly they decide the problem of compliance with the welding parameters and the accuracy of positioning of the welding torch. However, in these systems, insufficient attention is paid to the problem of operational control of the welding process in RTC on the criterion of product quality and prevention of unstable states.

These circumstances indicate the relevance of the development and implementation of new mathematical models and algorithms that allow to control the welding process robotic process systems on the criterion of quality of products, as well as to prevent the unstable operation of the RTC.

\footnotetext{
* Corresponding author: dm fominyh@mail.ru
} 


\section{Statement of the problem}

Taking into account experience of RTC operation, it is possible to define the main indicators of technological process influencing quality of the made production. The highest quality of production is reached at the minimum deviation of values of these indicators from the set values. Then the statement of the problem is as follows: to develop mathematical models and algorithms that allow to find the vector of control actions $\mathbf{p}(\mathrm{t}) \in\{\mathbf{P}\}$ on the time interval $\left[t_{\mathrm{P}}, t_{\mathrm{F}}\right]$, minimizing the target function:

$$
Q(t)=\sum_{i=1}^{n}\left(X_{i}^{*}(t)-X_{i}(t)\right)^{2} \omega_{\mathrm{i}} \rightarrow \min
$$

where $X_{i}(t), i=1,2, \ldots, n$ is factual quality indicators of the welding process in the RTC; $X_{i}^{*}(t)$ is the set value of indicator $X_{i}, \omega_{i}$ is weight coefficient of the $i$-th indicator. When solving the problem of minimizing the function $Q(t)$, it is important to exclude the system from entering a chaotic state. To do this, we introduce a constraint:

$$
\forall j\left(\rho_{j}\left(\mathbf{s}, \mathbf{s}_{j}^{*}\right)>\varepsilon\right), j=1,2, \ldots, m
$$

where $\mathbf{s}=\left\{X_{1}(t), X_{2}(t), \ldots, X_{n}(t)\right\}$ is the vector of the current system state, $\mathbf{s} \in \mathbf{S} ; \mathbf{S}^{*}-$ set of vectors of possible system states, $\mathbf{S}^{*}$ is the subset of state vectors in which the system goes unstable, $\mathbf{S}^{*} \subset \mathbf{S}, \mathbf{s}^{*} \in{ }_{j} \in \mathbf{S}^{*} ; \rho-$ a metric that defines the distance between two states in the space $\mathbf{S} ; \varepsilon$ is the specified constant.

\section{Mathematical model}

Due to the complexity of the control object, the use of methods of calculus of variations to solve the problem (1)-(2) is difficult. Therefore, to describe the relationship between the elements of the welding process in RTC the model of system dynamics was chosen [7-8]. The system dynamics model allows to construct differential equations of the following type for the main phase variables (system levels):

$$
d y(t) / d t=y^{+}(t)-y^{-}(t)
$$

where $y^{+}(t)$ is the positive rate of the variable $y(t)$, including all the factors that cause the growth of the variable $y(t) ; y^{-}(t)$ is the negative rate of variable $\mathrm{y}$, including all factors that cause the decrease of the variable $y(t)$.

The following indicators of the welding process in RTC are identified as system levels:

$X_{1}$ is the number of defective beams per 100 units of production; $X_{2}$ is the quantity of RTC operators; $X_{3}$ is the average number of stops of RTC per cycle; $X_{4}$ is average length of defective welds per 1 unit of production; $X_{5}$ is fulfilled works on scheduled maintenance of RTC; $X_{6}$ is the quantity of programmers; $X_{7}$ is the quantity of adjusters of welding equipment; $X_{8}$ is the quantity of QC inspectors; $\mathrm{X}_{9}$ is quantity of workshop technologists; $X_{10}$ is the number of days of delay in the supply of materials and spare parts for repairing RTC; $X_{11}$ is average deviation of welding arc voltage; $X_{12}$ is average current deviation on the engine of the wire feed unit; $X_{13}$ is the average deviation of the manipulator from the programmed trajectory; $X_{14}$ is presence in the workplace of the necessary technological documentation; $X_{15}$ is deflection of shielding gas pressure; $X_{16}$ is deflection of compressed air pressure; $X_{17}$ is production plan for a set period, in units of product; $X_{18}$ is the number of beams adopted by the QC inspectors from the first presentation. 
In addition, the model includes external factors that affect the above characteristics:

$O_{0}$ is the number of RTC operators at the beginning of the period; $O_{i n}$ is number of recruited RTC operators for the period; $O_{\text {out }}$ is the number of dismissed RTC operators for the period; $S_{m}$ is number of production shifts; $R_{w}$ is the number of RTC involved in the production process; $N_{s t}$ is the number of RTC stops per period; $S *$ is permissible number of RTC stops per welding cycle; $L_{d}$ is total length of defective weld seams for the period; $L^{*}$ is estimated length of defective weld seams for the period; $M_{f}$ is the number of completed activities of the scheduled preventive maintenance of RTC; $M_{p}$ is the number of planned activities of the scheduled preventive maintenance of RTC; $P_{0}$ is the quantity of programmers at the beginning of the period; $P_{i n}$ is the quantity of recruited programmers for the period; $P_{\text {out }}$ is the quantity of laid-off programmers for the period; $R_{0}$ is the quantity of adjusters of welding equipment at the beginning of the period; $R_{i n}$ is the quantity of recruited adjusters of welding equipment for the period; $R_{\text {out }}$ is the quantity of dismissed adjusters of welding equipment for the period; $C_{0}$ is the quantity of QC inspectors at the beginning of the period; $C_{\text {in }}$ is the quantity of recruited QC inspectors for the period; $C_{\text {out }}$ is the quantity of dismissed QC inspectors for the period; $T_{0}$ is the quantity of workshop technologists at the beginning of the period; $T_{\text {in }}$ is the quantity of recruited workshop technologists for the period; $T_{\text {out }}$ is the quantity of dismissed workshop technologists for the period; $N_{r}$ is the duration of repair of RTC; $D_{f}$ is the actual delivery time of spare parts and materials for repair of the RTC; $D_{p}$ is the planned delivery time of spare parts and materials for repair of RTC; $\Delta_{U}$ is the average deviation of the welding arc voltage from the nominal value; $\Delta^{*}{ }_{U}$ is the permissible deviation of the welding arc voltage from the nominal value; $\Delta_{I}$ is the average deviation of the current on the motor of the wire feed unit from the nominal value; $\Delta_{I}^{*}$ is the permissible current deviation on the motor of the wire feed unit from the nominal value; $\Delta T$ is the average deviation of the manipulator from the programmed trajectory; $\Delta^{*} T$ is the permissible deviation of the manipulator from the programmed trajectory; $T d_{f}$ is the actual number of documents of the technological process; $T d_{p}$ is the the required number of documents of the technological process; $\Delta_{P G}$ is the average deviation of the pressure of shielding gas ; $\Delta^{*}{ }_{P G}$ is the permissible deviation of the pressure of shielding gas ; $\Delta_{P V}$ is the average deviation of the pressure of compressed air; $\Delta^{*}{ }_{P V}$ is the permissible deviation of compressed air pressure; $N_{T P}$ is the number of beams assembled in accordance with the technological process; $N_{d}$ is the number of beams adopted to QC inspectors from the first presentation for the period; $A_{b}$ is the number of acts on nonconforming products for the period.

For the variable $X_{1}$, the differential equation (3) has the form:

$$
d X_{1}(t) / d t=\left(N_{W} f_{1}\left(X_{3}\right) f_{2}\left(X_{11}\right) f_{3}\left(X_{12}\right) f_{4}\left(X_{13}\right)\right)-\left(N_{S} f_{5}\left(X_{2}\right) f_{6}\left(X_{8}\right) f_{7}\left(X_{17}\right)\right)
$$

Equations for other variables are compiled in a similar way. As a result, the system of equations based on the mathematical model of system dynamics will look as follows: 


$$
\left\{\begin{array}{l}
d X_{1}(t) / d t=N_{W} f_{1}\left(X_{3}\right) f_{2}\left(X_{11}\right) f_{3}\left(X_{12}\right) f_{4}\left(X_{13}\right)-N_{S} f_{5}\left(X_{2}\right) f_{6}\left(X_{8}\right) f_{7}\left(X_{17}\right) \\
d X_{2}(t) / d t=\left(O_{0}+O_{i n}\right) f_{12}\left(X_{17}\right)-\left(S m+R w+O_{\text {out }}\right) \\
d X_{3}(t) / d t=N_{s t} / N_{W} f_{8}\left(X_{10}\right) f_{9}\left(X_{15}\right) f_{10}\left(X_{16}\right)-S^{*} f_{11}\left(X_{2}\right) \\
d X_{4}(t) / d t=L d f_{13}\left(X_{15}\right) f_{14}\left(X_{16}\right)-L^{*} f_{15}\left(X_{2}\right) \\
d X_{5}(t) / d t=M_{f} f_{16}\left(X_{6}\right) f_{17}\left(X_{7}\right)-M_{p} f_{18}\left(X_{10}\right) \\
d X_{6}(t) / d t=\left(P_{0}+P_{\text {in }}\right) f_{19}\left(X_{17}\right)-\left(S m+R w+P_{\text {out }}\right) \\
d X_{7}(t) / d t=\left(R_{0}+R_{\text {in }}\right) f_{20}\left(X_{17}\right)-\left(S m+R w+R_{\text {out }}\right) \\
d X_{8}(t) / d t=\left(C_{0}+C_{\text {in }}\right) f_{21}\left(X_{17}\right)-\left(S m+R w+C_{\text {out }}\right) \\
d X_{9}(t) / d t=\left(T_{0}+T_{\text {in }}\right) f_{22}\left(X_{17}\right)-T_{\text {out }} \\
d X_{10}(t) / d t=\left(N r+D_{f}\right) f_{23}\left(X_{17}\right)-D_{p} \\
d X_{11}(t) / d t=\Delta_{U}-\Delta_{U}^{*} f_{24}\left(X_{5}\right) \\
d X_{12}(t) / d t=\Delta_{I}-\Delta_{I}^{*} f_{25}\left(X_{5}\right) \\
d X_{13}(t) / d t=\Delta_{T}-\Delta_{T}^{*} f_{26}\left(X_{5}\right) \\
d X_{14}(t) / d t=T d_{f} f_{27}\left(X_{9}\right)-T d_{p} \\
d X_{15}(t) / d t=\Delta_{P G}-\Delta_{P G}^{*} f_{28}\left(X_{17}\right) \\
d X_{16}(t) / d t=\Delta_{P V}-\Delta_{P V}^{*} f_{29}\left(X_{17}\right) \\
d X_{17}(t) / d t=N_{T P} f_{30}\left(X_{9}\right)-N_{W} \\
d X_{18}(t) / d t=N_{d} f_{31}\left(X_{6}\right) f_{32}\left(X_{7}\right) f_{33}\left(X_{8}\right) f_{34}\left(X_{14}\right)-(A b+L d) f_{35}\left(X_{1}\right) f_{36}\left(X_{4}\right)
\end{array}\right.
$$

where $f\left(X_{i}\right)$ is the functional dependence on indicator $X_{i}$, obtained as a result of polynomial approximation of statistical data.

Due to the high dimensionality and nonlinearity, it is difficult to obtain an analytical solution of the system of equations (5), so the values of indicators $X_{1}, X_{2}, \ldots, X_{18}$ are determined by its numerical solution.

Further, to solve the problem (1), it is necessary to find the vector of control actions to minimize the function $Q(t)$. Control actions are implementing in the form of activities plan $\mathbf{p}_{\mathrm{j}} \in\{\mathbf{P}\}, j=1,2, . ., N$ :

By calculating the values of the $Q(t)$ function for each plan $\mathbf{p}_{\mathrm{j}} \in\{\mathbf{P}\}$ on a given time interval, we can define an action plan which implementation minimizes the target function and is therefore a solution to the problem (1).

\section{Identification of chaotic states}

To fulfill the condition (2) it is necessary to develop an algorithm for identification of unstable states. Determine in advance all the possible unstable states is not possible, so we take as a basis one of the most common types of chaos - the Rossler attractor. During the further operation of the system, the set of possible unstable states will be replenished.

Let's define the values of the main indicators of quality $X_{1}$ (the number of defective beams per 100 units of production), $X_{4}$ (average length of defective welds per 1 unit of production) and $X_{18}$ (the number of beams adopted by the QC inspectors from the first presentation), in which the system (5) takes the form of a Rossler's system of equations: 


$$
\left\{\begin{array}{l}
d X_{1}(t) / d t=-X_{4}(t)-X_{18}(t) \\
d X_{4}(t) / d t=X_{1}(t)+a X_{4}(t) \\
d X_{18}(t) / d t=b+X_{18}(t)\left(X_{1}(t)-c\right)
\end{array}\right.
$$

when $\mathrm{a}=\mathrm{b}=0,2 ; \mathrm{c}=5,7$.

$$
\left\{\begin{array}{l}
X_{4}(t)+X_{18}(t)+N_{W} X_{3}(t)\left(1-X_{12}(t)\left(X_{13}(t)-X_{11}(t)+1\right)\right)-N_{S}\left(X_{8}(t)-2 X_{17}(t)-\right. \\
\left.-X_{2}(t)\left(1-X_{17}(t)\right)+3\right)=0 \\
L d\left(0,247-0,081 X_{15}(t)+X_{16}(t)\left(0,268-0,088 X_{15}(t)\right)\right)-L^{*}\left(0,914-0,473 X_{2}(t)\right)- \\
-X_{1}(t)-0,2 X_{4}(t)=0 \\
(A b+L d)\left(1,28-0,66 X_{4}(t)+X_{1}(t)\left(0,67 X_{4}(t)-1,3\right)\right)-N_{d}\left(16 X_{14}(t)+X_{7}(t)\left(X_{14}(t)+\right.\right. \\
\left.\left.+20 X_{8}(t)+87\right)-X_{6}(t)\left(X_{7}(t)+3 X_{8}(t)+12\right)+4 X_{8}(t)\left(X_{14}(t)+29\right)+518\right)- \\
-X_{1}(t) X_{18}(t)-5,7 X_{18}(t)=0,2
\end{array}\right.
$$

The existence of a nonzero solution of the system of equations (6) will be a necessary condition for the existence of an unstable state. A positive value of the highest Lyapunov exponent will be a sufficient condition for the system to be chaotic [9].

The found solutions of the system of equations (6) are entered into the database of unstable states of the system. In solving the problem (1) control actions are selected so that the system does not fall into one of the unstable states. The database of unstable states can be replenished using other types of chaos: Lorenz, Nose-Hoover attractors, etc.

Thus, the solution of problem (1)-(2) consists of the following stages:

- Construct the mathematical model (4) describing technological process of welding in RTC and allowing to calculate values of $Q(t)$,

- Determine the vector of control actions in the form of an activities plan that minimizes the function $Q(t)$,

- Form the state vector $\mathbf{s}^{+}$, , to which the system will go after the selected control action,

- Find the distance from $\mathbf{s}^{+}$to the nearest unstable state $\mathbf{s}^{*}{ }_{\mathrm{j}} \in \mathbf{S}^{*}$ via metric:

$$
\rho\left(\mathbf{s}_{\mathrm{i}}, \mathbf{s}_{\mathrm{j}}\right)=\sqrt{\sum_{\mathrm{k}=1}^{\mathrm{n}}\left(\omega_{\mathrm{i}}\left(\mathrm{X}_{\mathrm{k}}^{(\mathrm{i})}-\mathrm{X}_{\mathrm{k}}^{(\mathrm{j})}\right)^{2}\right)}
$$

- If the condition (2) is satisfied, the problem was solved, otherwise it is necessary to return to step 2 to select the control action vector, excluding the already selected vector.

\section{Conclusion}

The statement of a problem of management of process of welding in RTC in the conditions of risk of emergence of unstable states is developed in this article. The welding process parameters affecting the quality of products are selected, the mathematical model of system dynamics which allows to determine their changes in a given time interval is developed.

The algorithm of identification of chaotic states on the example of the attractor Ressler is also offered. The procedure of prevention chaotic conditions is developed. Currently, the developed mathematical software undergo testing on the basis of a complex of technical means of control RTC Kawasaki at OJSC "Transmash "(Engels, Russia) by methods [10$16]$. 


\section{References}

1. V. Filaretov, Self-tuning control systems of manipulators actuators, Vladivostok: FESTU, 2000 (in Russian)

2. V. Filaretov, D. Yukhimet, A. Zuev, A. Gubankov, Bulletin of the Far Eastern Branch of the Russian Academy of Sciences, 4 (2016) (in Russian)

3. D. Fominykh, V. Kushnikov, Proceedings of XXX International Scientific Conference Mathematical Methods in Technique and Technology - MMTT, 6 (2017) (in Russian)

4. A. Rezchikov, V. Kushnikov, V. Ivashchenko, D. Fominykh, A. Bogomolov, L. Filimonyuk, Journal of machinery manufacture and reliability, 4, 46 (2017)

5. A. Rezchikov, V. Kushnikov, V. Ivashchenko, D. Fominykh, A. Bogomolov, L. Filimonyuk, Mechatronics. Automation. Control, 5 (2017) (in Russian)

6. A. Rezchikov, V. Kushnikov, V. Ivashchenko, D. Fominykh, A. Bogomolov, L. Filimonyuk, Proceedings of Voronezh State University. Series: systems analysis and information technologies, 4 (2017) (in Russian)

7. J.W. Forrester, Industrial Dynamics, MIT Press, Cambridge, Mass, 1961

8. A. Rezchikov, V. Kushnikov, V. Ivashchenko, D. Fominykh, A. Bogomolov, L. Filimonyuk, Large-scale systems control, 71 (2018) (in Russian)

9. B. Andrievsky, A. Fradkov, Automation and remote control, 4, 5 (2003)

10. D. Fominykh, E. Shlychkov, V. Kushnikov, A. Rezchikov, A. Sankova, Automation. Modern technologies, 12 (2007) (in Russian)

11. V. Kushnikov, A. Rezchikov, A. Tsvirkun, Automation and Remote Control, 59, 7, 2 (1998)

12. V. Kushnikov, A. Rezchikov, A. Tsvirkun, Meitan Kexun Jishu, 11, 26 (1998)

13. A. Rezchikov, O. Dolinina, V. Kushnikov, V. Ivashchenko, K. Kachur, A. Bogomolov, L. Filimonyuk, Indian Journal of Science and Technology, 9, 46 (2016)

14. A. Rezchikov, V. Kushnikov, V. Ivashchenko, D. Fominykh, A. Bogomolov L. Filimonyuk, Bulletin of the Astrakhan State Technical University. Series: management, computer technology and informatics, 4 (2017) (in Russian)

15. A. Rezchikov, V. Kushnikov, V. Ivashchenko, D. Fominykh, A. Bogomolov L. Filimonyuk, Control Systems and Informational Technologies, 3, 69 (2017) (in Russian)

16. E. Shlychkov, D. Fominykh, V. Kushnikov, A. Rezchikov, Automation. Modern technologies, 8 (2006) (in Russian) 